\title{
Associação entre Diferentes Indicadores de Obesidade e Prevalência de Hipertensão Arterial
}

\author{
M iguel Gus, Leila B. Moreira, Maurício Pimentel, A na Luiza M. Gleisener, Renan S. Moraes, \\ Flávio D. Fuchs \\ PortoAlegre, RS
}

Objetivo - Determinar a associação entre índice de massa corporal (IMC), índice cintura-quadril e cintura com a prevalência de hipertensão arterial (HAS) em amostra representativa de 1088 adultos de Porto Alegre, Brasil.

Métodos - Foram considerados hipertensos indivíduos com pressão sistólica $(P A S) \geqslant 160 \mathrm{mmHg}$ ou diastólica $(P A D) \geqslant 95 \mathrm{mmHg}$ e definidos como obesos aqueles com IMC $\geqslant 27 \mathrm{~kg} / \mathrm{m}^{2}$, ou com razão cintura/quadril $\geqslant 0,95$ (homens) $e \geqslant 0,80$ (mulheres) ou com cintura $\geqslant 96 \mathrm{~cm}$ (homens) $e \geqslant 92 \mathrm{~cm}$ (mulheres).

Resultados - A obesidade aferida pelo IMC associouse com a prevalência de HAS em ambos sexos (RR 1,9, IC 1,0 - 3,2 masculino; RR 2,2, IC 1,3 - 3,8 feminino). Os outros indices associaram-se, significativamente, apenas nas mulheres.

Conclusão- $I M C \geqslant 27,0 \mathrm{~kg} / \mathrm{m}^{2}$ associou-se mais, consistentemente, com o risco de HAS. A magnitude similar das associações dos demais indicadores demonstram sua utilidade na avaliação do risco para hipertensão.

Palavras-chave: hipertensão, obesidade, prevalência

\section{TheAssociation between Different Measure- ments of Obesity and the Prevalence of $\mathrm{Hy}$ - pertension}

Purpose - To evaluate the association between body mass index (BMI), waist-hip ratio and waist circumference with the prevalence of hypertension in a representative sample of 1088 adults of Porto Alegre (RS), Brazil.

Methods - In this cross-sectional survey, subjects were considered as having hypertension if they had systolic blood pressure $\geqslant 160 \mathrm{mmHg}$ or diastolic $\geqslant 90 \mathrm{mmHg}$, and were considered obese if they had $B M I \geqslant 27 \mathrm{~kg} / \mathrm{m}^{2}$, or had a waist-hip-ratio $\geqslant 0.95 \mathrm{~cm}$ (men) or 0.80 (women) or had a waist circumference $\geqslant 96$ (men) or 92 (women).

Results - Obesity defined by the BMI was associated with hypertension in both genders (RR 1.9, CI 1.0 - 3.2 in men; RR 2.2, CI 1.3 -3.8 in women). The other indices were significantly associated with hypertension just in women.

Conclusion - BMI $\geqslant 27.0 \mathrm{~kg} / \mathrm{m}^{2}$ was strongly associated with increased odds to have hypertension. Similar magnitude of the association with the other indices indicate their utility to estimate the risk of hypertension.

Key-words: hypertension, obesity, prevalence

Arq Bras Cardiol, volume 70 (n' 2$)$, 111-114, 1998

A obesidade é um fator de risco independente para hipertensão arterial sistêmica (HAS) ${ }^{1,2,3}$ e para mortalidade, especialmente em mulheres ${ }^{5,6}$. Dieta hipocalórica tem sido considerada uma medida eficaz ${ }^{4}$ e efetiva ${ }^{6}$ no manejo da HAS.

O índice de massa corporal (IMC) e a razão cinturaquadril são as medidas antropométricas mais comumente

Unidade de Farmacologia Clínica do Hospital de Clínicas de Porto Alegre e Depto de Farmacologia, Universidade Federal do Rio Grande do Sul Correspondência: Miguel Gus - Rua Padre Chagas, 25 - 90570-080 - Porto Alegre, RS

Recebido para publicação em 23/7/97

Aceito em 3/12/97 utilizadas para a determinação do excesso de peso, estando, ainda, associadas a outros fatores de risco cardiovasculares, como hipercolesterolemia, baixos níveis de lipoproteína de alta densidade (HDL) e resistência insulínica ${ }^{8}$.

Diversos índices antropométricos têm sido propostos para determinar a associação entre excesso de peso e fatores de risco cardiovasculares. A medida da cintura, um dado de fácil e rápida obtenção, associa-se tanto com o IMC como com a razão cintura-quadril, sendo capaz de detectar indivíduos com excesso de peso e outros fatores de risco cardiovascular com alta acurácia ${ }^{9-11}$. A medida da cintura também está relacionada com a gordura intra-abdominal e já 
foi sugerida como o índice antropométrico capaz de prever o risco cardiovascular ${ }^{12}$.

O presente trabalho tem o objetivo de detectar qual o índice antropométrico que se associa mais significativamente com a prevalência de HAS e em qual deles um aumento importante estaria relacionado a maior prevalência de HAS, em uma amostra de indivíduos adultos não selecionados por doença e representativa da população de Porto Alegre.

\section{Métodos}

Selecionaram-se 1088 indivíduos com idade mínima de 18 anos através de processo de amostragem aleatória proporcional por estágios múltiplos e conglomerados. Os dados foram coletados em visita domiciliar por entrevistadores treinados para a aplicação de um questionário padronizado e aferição dos parâmetros físicos, incluindo a pressão arterial (PA). A descrição completa da amostragem e da coleta dos dados já foi publicada ${ }^{2,12,14}$.

A PA foi aferida com manômetros aneróides, periodicamente calibrados, contra manômetros de mercúrio e seguindo as orientações do Joint National Committee ${ }^{15}$. Considerou-se o $1^{\circ}$ som de Korotkoff como a pressão arterial sistólica (PAS) e o 5ำ como pressão arterial diastólica (PAD). Para efeito de análise foi considerada a média de duas aferições, com correção para o perímetro braquial, segundo as fórmulas propostas por Maxwell ${ }^{16}:$ PAS = sistólica aferida + $[32-(1,05 \times$ perímetro braquial $)] \mathrm{e} \mathrm{PAD}=$ diastólica aferida + [22- $(0,72 \times$ perímetro braquial $)]$.

Verificou-se o peso corporal dos indivíduos vestindo roupas leves e descalços utilizando-se balanças portáteis com precisão de $0,1 \mathrm{~kg}$. Aferiram-se a altura, a circunferência braquial, a cintura e quadril com fita métrica e a altura com trenas.

Calculou-se IMC como peso dividido pela altura ao quadrado $\left(\mathrm{kg} / \mathrm{m}^{2}\right)$. A medida da cintura foi realizada na porção de menor circunferência entre o gradil costal e a crista ilíaca e a medida do quadril ao nível dos grandes trocânteres.

Utilizando-se como padrão ouro para determinação do excesso de peso um IMC $\geqslant 27 \mathrm{~kg} / \mathrm{m}^{2}$ determinou-se através de curva ROC (receiver operating characteristics) ${ }^{11} \mathrm{O}$ ponto de corte da medida da cintura em $96 \mathrm{~cm}$ para homens e
$92 \mathrm{~cm}$ para mulheres, como indicadores de excesso de peso. Com base em outros estudos ${ }^{8,9}$, estabeleceu-se o ponto de corte para a razão cintura-quadril em 0,95 para homens e 0,80 para mulheres.

Consideraram-se hipertensos os indivíduos com PAS $\geqslant 160 \mathrm{mmHg}$ e/ou PAD $\geqslant 95 \mathrm{mmHg}^{17}$, tomando-se a média de duas aferições corrigidas pelo perímetro braquial ${ }^{16}$.

A associação entre os diferentes índices antropométricos e a prevalência de HAS foi analisada através de regressão logística. As variáveis de controle foram idade, história familiar de hipertensão (ambos os pais hipertensos), abuso de álcool (consumo $\geqslant 30 \mathrm{~g}$ ) e alcance escolar.

Os cálculos estatísticos foram realizados utilizando-se o programaSPSS ${ }^{16}$.

\section{Resultados}

A média de idade, IMC e razão cintura-quadril e a prevalência de HAS foram similares para homens e mulheres. A cintura foi significativamente maior nos homens (tab. I). Os homens apresentaram PAS maior do que as mulheres, assim como maior prevalência de abuso de álcool e maior frequiência de escolaridade $<5$ anos, variáveis que nesta amostra associaram-se, de maneira independente, com a prevalência de HAS ${ }^{2}$.

\begin{tabular}{|lcc|}
\hline \multicolumn{2}{|c|}{$\begin{array}{c}\text { Tabela I - Características da amostra segundo o sexo (média e desvio } \\
\text { padrão ou N e percentagem, quando cabível) }\end{array}$} \\
\hline Características & Homens (n=488) & Mulheres (n=600) \\
\hline Idade (anos) & $41,4 \pm 16,1$ & $43,9 \pm 17,3$ \\
Peso (kg) & $73,7 \pm 13,4$ & $63,0 \pm 13,2$ \\
Altura (m) & $1,71 \pm 0,05$ & $1,57 \pm 6,6$ \\
IMC (kg/m²) & $25,0 \pm 4,1$ & $25,2 \pm 5,1$ \\
Razão cintura-quadril & $0,88 \pm 0,08$ & $0,80 \pm 0,08$ \\
Cintura (cm) & $89 \pm 11 *$ & $82 \pm 12$ \\
Predisposição familiar para hipertensão & $47(9,6)$ & $68(11,3)$ \\
Abuso de álcool & $143(29,3)^{*}$ & $25(4,2)$ \\
Escolaridade <5 anos & $130(26,6)^{*}$ & $76(12,7)$ \\
PAS (mmHg) & $129 \pm 20^{*}$ & $125 \pm 25$ \\
PAD (mmHg) & $78 \pm 13$ & $75 \pm 15$ \\
HAS ( $\geqslant 160 / 95 m m ~ H g)(n ~ e ~ \%) ~$ & $65(13,3)$ & $70(11,7)$ \\
\hline
\end{tabular}

IMC- índice de massa corporal; PAS- pressão arterial sistólica; PAD- pressão arterial diastólica; HAS- hipertensão arterial sistêmica; * $\mathrm{p}<0,05$.

Tabela II - Diferenças entre PAS, PAD, prevalência de hipertensão arterial (HAS) e peso entre os homens com ou sem excesso de peso definidos pelo índice de massa corporal (IMC), razão cintura-quadril (C/Q) e cintura

\begin{tabular}{|c|c|c|c|c|c|c|}
\hline & \multicolumn{2}{|c|}{$\operatorname{IMC}\left(\mathrm{kg} / \mathrm{m}^{2}\right)$} & \multicolumn{2}{|c|}{ Cintura/Quadril } & \multicolumn{2}{|c|}{ Cintura $(\mathrm{cm})$} \\
\hline & $(\geqslant 27)$ & $(<27)$ & $(\geqslant 0,95)$ & $(<0,95)$ & $(\geqslant 96)$ & $(<96)$ \\
\hline PAS (mmHg) & $137 \pm 23^{*}$ & $125 \pm 17$ & $141 \pm 25^{*}$ & $126 \pm 17$ & $137 \pm 24 *$ & $126 \pm 17$ \\
\hline $\mathrm{PAD}(\mathrm{mmHg})$ & $85 \pm 16^{*}$ & $76 \pm 11$ & $86 \pm 15$ & $77 \pm 12$ & $85 \pm 15$ & $76 \pm 12$ \\
\hline $\operatorname{HAS}(\%)$ & $22,6^{*}$ & 9,9 & $27,6^{*}$ & 9,77 & $23,8 *$ & 9,7 \\
\hline Peso $(\mathrm{kg})$ & $88,3 \pm 12 *$ & $68,2 \pm 8,6$ & $81,3 \pm 16,2 *$ & $71,7 \pm 11,3$ & $87,8 \pm 13,4 *$ & $68,7 \pm 9$ \\
\hline Idade (anos) & $48,3 \pm 14,6$ & $38,8 \pm 15,9$ & $54,9 \pm 12 *$ & $38,0 \pm 15^{*}$ & $51,7 \pm 14$ & $37,8 \pm 15$ \\
\hline
\end{tabular}


Tabela III - Diferenças entre PAS, PAD, prevalência de hipertensão arterial e peso entre as mulheres com ou sem excesso de peso definidos pelo índice de massa corporal (IMC), razão cintura-quadril (C/Q) e cintura

\begin{tabular}{|c|c|c|c|c|c|c|}
\hline & \multicolumn{2}{|c|}{$\mathrm{IMC}\left(\mathrm{kg} / \mathrm{m}^{2}\right)$} & \multicolumn{2}{|c|}{ Cintura/Quadril } & \multicolumn{2}{|c|}{ Cintura $(\mathrm{cm})$} \\
\hline & $(\geqslant 27)$ & $(<27)$ & $(\geqslant 0,80)$ & $(<0,80)$ & $(\geqslant 92)$ & $(<92)$ \\
\hline $\mathrm{PAD}(\mathrm{mmHg})$ & $82 \pm 17 *$ & $72 \pm 13$ & $80 \pm 16^{*}$ & $71 \pm 13$ & $84 \pm 17 *$ & $73 \pm 13$ \\
\hline HAS (\%) & $20,9^{*}$ & 7,9 & $18,4 *$ & 5,9 & $24,2 *$ & 8,5 \\
\hline Peso (kg) & $77,2 \pm 13,8^{*}$ & $57,3 \pm 7,5$ & $66,5 \pm 13,0^{*}$ & $60,0 \pm 12,2$ & $79,0 \pm 15,6^{*}$ & $59,0 \pm 9,8$ \\
\hline Idade (anos) & $51,4 \pm 16^{*}$ & $40,9 \pm 16,7$ & $50,9 \pm 17,3 *$ & $37,9 \pm 15,4$ & $53,5 \pm 16,2 *$ & $41,5 \pm 16,4$ \\
\hline
\end{tabular}

PAS- pressão arterial sistólica; PAD- pressão arterial diastólica; HAS- hipertensão arterial sistêmica; * p<0,05 para a comparação do parâmetro em cada indicador de obesidade.

\begin{tabular}{|c|c|c|c|c|c|c|}
\hline \multirow{5}{*}{$\begin{array}{l}\text { Cintura } \\
\mathrm{IMC} \\
\mathrm{C} / \mathrm{Q}\end{array}$} & \multicolumn{3}{|c|}{ Homens } & \multicolumn{3}{|c|}{ Mulheres } \\
\hline & Beta & $\mathrm{P}$ & $\operatorname{Exp} B$ (IC) & Beta & $\mathrm{P}$ & $\operatorname{Exp} B(I C)$ \\
\hline & 0,51 & 0,08 & $1,7(0,9-2,9)$ & 0,84 & 0,003 & $2,3(1,3-4,1)$ \\
\hline & 0,61 & 0,03 & $1,9(1,0-3,2)$ & 0,79 & 0,005 & $2,2(1,3-3,8)$ \\
\hline & 0,58 & 0,06 & $1,7(0,97-3,2)$ & 0,70 & 0,02 & $2,2(1,1-3,6)$ \\
\hline
\end{tabular}

A tabela II e III mostram as médias das PAS e PAD, prevalência de HAS e peso entre homens e mulheres come sem excesso de peso definido pelos diferentes índices antropométricos. A PAS e a prevalência de HAS foram mais elevadas entre os homens com excesso de peso determinado por qualquer um dos índices antropométricos. A PAD foi significativamente mais elevada apenas entre os indivíduos com excesso de peso definido pelo IMC. Entre as mulheres, a PAS, PAD e a prevalência de HAS foram significativamente mais elevadas entre as com excesso de peso, determinado por qualquer um dos índices antropométricos.

Os riscos relativos estimados de HAS, ajustados para idade, história familiar de hipertensão, abuso de álcool e escolaridade são apresentados na tabela IV. O excesso de peso definido por IMC $\geqslant 27 \mathrm{~kg} / \mathrm{m}^{2}$ apresentou associação estatisticamente significativa com a prevalência de HAS, tanto em homens (1,9 IC 1,0-3,2) como em mulheres (2,2 IC 1,3-3,8). A medida da cintura e a razão cintura-quadril apresentaram associação estatisticamente significativa apenas entre as mulheres (2,3 IC 1,3-4,1 e 2,2 IC 1,1-3,6, respectivamente).

\section{Discussão}

Os primeiros estudos sobre a composição corporal e distribuição da gordura corporal para identificar indivíduos com um risco maior para doença cardiovascular e diabetes mellitus tipo II utilizaram, principalmente, o IMC e a razão cintura-quadril ${ }^{19,20}$. A medida da cintura é um índice antropométrico de fácil e rápida aferição, dispensando a necessidade do emprego de fórmulas e cálculos.

Han e col ${ }^{10,11}$ demonstraram, em estudo transversal e de base populacional, que a medida da cintura é capaz de identificar com alta acurácia indivíduos obesos. Estes autores identificaram que circunferências $\geqslant 94 \mathrm{~cm}$ em homens e $\geqslant 80 \mathrm{~cm}$ em mulheres são capazes de identificar indivíduos com IMC $\geqslant 25 \mathrm{~kg} / \mathrm{m}^{2}$ com uma sensibilidade e especificidade de $96 \%$. Além disto verificaram uma associação significativa entre a medida de cintura acima destes valores e a prevalência de hipertensão e outros fatores de risco, tanto em homens quanto em mulheres.

No presente estudo, igualmente, foi identificada uma associação positiva entre os diferentes índices antropométricos indicadores de obesidade e a prevalência de hipertensão. Em relação à cintura, as diferenças nos valores do risco relativo estimado no estudo de Han e col ${ }^{10,11}$ para o estudo atual (1,9 vs 1,7 e 1,8 vs 2,3 para homens e mulheres, respectivamente) poderiam ser explicadas pelas diferenças nos pontos de corte ou características da população.

No modelo de regressão logística do presente estudo, o IMC foi o indicador de obesidade com associação mais consistente com a prevalência de hipertensão, tanto em homens como em mulheres, representando um risco duas vezes maior para valores maiores ou iguais a $27 \mathrm{~kg} / \mathrm{m}^{2}$.

A importância do IMC como indicador de excesso de peso já foi demonstrada em estudos de coorte ${ }^{5,6}$ que avaliaram a relação entre mortalidade e obesidade. Nesses trabalhos foi demonstrado um aumento da mortalidade total associado a um IMC $>27 \mathrm{~kg} / \mathrm{m}^{2}$. Devido a isto, escolheu-se valor diferentemente do valor de $25 \mathrm{~kg} / \mathrm{m}^{2}$ adotado no estudo de Han e col ${ }^{10}$ no momento da identificação dos pontos de corte para a definição de obesidade, a partir da medida da cintura.

Embora não atingissem significância estatística, entre os homens, os resultados obtidos para os demais indicado- 
res de obesidade são coerentes, mostrando riscos relativos estimados de magnitudes semelhantes, o que sugere que a cintura possa ser utilizada em substituição ao IMC, inclusive para os homens. A razão cintura-quadril demonstrou associação praticamente semelhante ao IMC.

Este trabalho permite concluir que em uma amostra populacional de adultos, representativa da cidade de Porto Alegre, a medida de cintura $\geqslant 96 \mathrm{~cm}$ para homens e $\geqslant 92 \mathrm{~cm}$ para mulheres associa-se, significativamente, à obesidade definida como IMC $\geqslant 27 \mathrm{~kg} / \mathrm{m}^{2}$. O índice antropométrico que se associou mais consistentemente com a prevalência de HAS foi o IMC, mas a intensidade similar da associação entre o índice cintura/quadril e medida isolada da cintura demonstram que estes índices podem ser utilizados como opção durante a avaliação do risco de hipertensão e na avaliação de indivíduos hipertensos.

\section{Referências}

1. Whelton PK - Epidemiology of hypertension. Lancet 1994; 344:101-6.

2. Fuchs FD, Moreira LB, Moraes RS, Bredemeier M, Cardozo SC-Prevalência de hipertensão arterial sistêmica e fatores associados na região urbana de Porto Alegre. Estudo de base populacional. Arq Bras Cardiol 1995; 63: 473-9.

3. Gus M, Fuchs FD-Obesidade e hipertensão. Arq Bras Cardiol 1995; 64: 565-70.

4. Alderman MH - Non-pharmacological treatment of hypertension. Lancet 1994; 344:307-10.

5. Manson JE, Willett WC, Stampfer MJ et al - Body weight and mortality among women. N Engl J Med 1995; 333: 677-85.

6. Iribarren C, Sharp DS, Burchfiel CM, Petrovich $\mathrm{H}$ - Association of weight los and weight fluctuation with mortality among Japanese American men. N Engl J Med 1995; 333: 686-92.

7. FuchsFD, Gus M,MoreiraWD-Blood pressure effects of antihypertensive drugs and lifestyle modification in a Brazilian hypertensive cohort. J Hypert 1997 (no prelo).

8. Björntorp P - Classification of obese patients and complications related to the distribution of surplus fat. Am J Clin Nutr 1987; 45(supl 5): 1120-5.

9. Lean MEJ, Han TS, Morrison CE - Waist circumference as a measure for indicating need for weight management. BrMed J 1995; 311: 158-61.

10. Han TS, Van LeerEM, Seidell MEJ, Lean MEJ - Waist circumference action level in the identification of cardiovascular risk factors: prevalence study in a random sample. Br Med J 1995; 311: 1401-5.

11. Han TS, Van Leer EM, Seidell MEJ, Lean MEJ - Waist circumference as a screening tool for cardiovascular risk factors: evaluation of receiver operating characteristics (ROC). Obes Res 1996; 4: 533-47.

12. Pouliot MC, Després JP, Lemieux S et al - Waist circumference and abdominal sagittal diameter: best anthropometric indexes of abdominal visceral tissue accumu- lation and related cardiovascular risk in men and women. Am J Cardiol 1994; 73: 460-8.

13. Moreira LB, Fuchs FD, Moraes RS et al - Alcoholic consumption and associated factors in Porto Alegre, a Southern Brazilian city: a population based survey. J Std Alcohol 1996; 57: 253-9.

14. Moreira LB, Fuchs FD, Moraes RS, Bredemeier M, Cardoso S - Prevalência de tabagismo e fatores associados em área metropolitana da região sul do Brasil. Rev Saúde Pública 1995; 29: 46-51.

15. The Fifth Report of the Joint National Committee on Detection, Evaluation and Treatment of High Blood Pressure. Arch Intern Med 1993; 153: 154-83.

16. Maxwell MH, Waks AU, Schroth PC, Karam M, Dornfeld LP - Error in blood pressure measurement due to incorrect cuff size in obese patients. Lancet 1982;2: 33-5.

17. World Health Organization - Geographical variation in the major risk factors of coronary heart disease in men and women aged 35-64. The MONICA project. World Health Statistics Q.1988; 41: 115-37.

18. Norusis MJ - Base System User's Guide. SPSS for Windows: Release 6. Chicago: IL: SPSS, 1993.

19. Lapidus L, Bengtsson C, Larsson B, Pennert K, Rybo E, Sjöström L - Distribution of adipose tissue and risk of cardiovascular disease and death. 12 year follow-up of participants in the population study of women in Gothenburg, Sweden. Br Med J 1984; 289: 1261-3.

20. Larsson B, Svärdsudd K, Welin L, Wilhelmsen L, Björntorp P, Tibblin G - Abdominal adipose tissue distribution, obesity and risk of cardiovascular disease and death: 13 year follow-up of participants in the study of men born in 1913. Br Med J 1984; 228: 1401-4. 